\section{Working memory in children with Down's syndrome}

\section{Irene Broadley}

University of Southampton, U.K.

\section{John MacDonald and Sue Buckley}

University of Portsmouth, U.K.

\begin{abstract}
A group of 4 to 18 year old children with Down's syndrome $(\mathrm{N}=62)$ was presented with a set of working memory tasks, including auditory and visual serial recall of words; standardised digit span tasks and a rhyme judgement task. The serial recall tasks involved pictures of common objects or the spoken names of these objects and the children had to recall lists which varied on a number of parameters, including word length and the acoustic similarity of the object names. It was found that contrary to expectation the children's performance showed significant effects of word length and acoustic similarity, which are normally taken to indicate phonological storage and speech based rehearsal. These effects were found in both the auditory and visual presentation conditions and for the youngest age group. In addition to this evidence for speech based storage in short-term memory there was also evidence of the children utilising visual information in the serial recall tasks. The results are discussed in terms of working memory operation and the implications for memory remediation strategies in children with Down's syndrome.
\end{abstract}

\section{Acknowledgements}

This research was supported by a research bursary to Irene Broadley from the Portsmouth Down's Syndrome Trust. The authors would like to thank all the children, parents and teachers who participated in the study.

(C) 1993,1999. The Down Syndrome Educational Trust Down Syndrome Research and Practice 1995, 3 (1) 3-8
Introduction

The effort to explain the workings of short-term memory has led to a number of theoretical models. One of the more popular is the 'working memory' model developed by Baddeley and Hitch (1974). This three component model, comprising a central executive, phonological loop and visuospatial sketch pad has been found to account for a wide variety of experimental findings in normal individuals and clinical populations (Baddeley, 1986). More critically for the concerns of this paper, the model has also been used to explain variation in everyday cognitive activities. For example, Gathercole and Baddeley (1989), found that phonological memory skills in four and five year olds were directly related to vocabulary learning.

Furthermore, the model has been used to describe the development of short-term memory functioning (Hitch and Halliday, 1983). This strategy of applying the model to a wider set of memory phenomena and different subject populations not only allows the other areas a potentially powerful theoretical account, but also provides a test of the theoretical generality of the model. The subject of this paper is to apply the 'working memory' model to memory data obtained from a group of children with Down's syndrome, the important aspect being that this is a subject population known to have poor short-term memory performance in comparison to typically developing and mental age matched peers (Mackenzie and Hulme, 1987). If there is a close relationship between short-term memory functioning and performance on everyday cognitive tasks then identification of the underlying processes that might account for these deficits could provide a clue to either remediation or alternative teaching and learning strategies. The remainder of this section will briefly review the working memory model in a developmental context, outline what is known about memory in children with Down's syndrome and then derive some implications to be examined in the experiment described.

The current version of the 'working memory' model (Baddeley, 1986) proposes three components or subsystems. The first of these, the central executive, is basically an attentional system that controls and monitors the operation of the two other components. The second is the visuo-spatial sketch pad which stores visual and/or spatial information and material. The third which is of primary importance for the work described here is the phonological loop which is specialised for the storage of verbal information. This loop consists of two related but independent subcomponents, a phonological store and an articulatory loop. The phonological store can receive information from either the auditory system or from visually presented text information recoded into a speech based form. Information in the store decays rapidly unless it is recycled, which is the process carried out by the articulatory loop. This is hypothesised to be an active rehearsal based process, possibly involving subvocal articulation. This system is presumed to have a limited capacity which is time based, i.e. it stores approximately 2 seconds worth of verbal material.

The phonological loop mechanism accounts for a number of standard findings from tasks involving the short-term storage and recall of verbal information, for example the word length and acoustic similarity effects. The word length effect is the poorer recall for lists of long words than lists of short words. This appears to be the result of the time limited nature of the rehearsal process; it takes longer to articulate 
words with more syllables therefore fewer can be placed in the loop (Baddeley, Thomson and Buchanan, 1975). On the other hand, the acoustic similarity effect - poorer recall of lists of phonologically similar items than lists of dissimilar items, is due to the coding of information in the phonological store. Poorer recall results from the difficulty of discriminating between decaying memory traces which are similar. Since the items are presented verbally the coding must be based on speech sounds (Salamé and Baddeley, 1986).

The development of short-term memory capability has had considerable research attention over recent years. In normally developing children verbal memory span tested with either digits or words as stimuli increases from approximately 2 to 3 items at 4 years of age to 7 to 8 items at 14 years of age (Chi, 1977). The explanation for this change from a working memory perspective is that the younger children have slower rates of articulation, which means slower and less efficient subvocal rehearsal therefore fewer items can be retained in the articulatory loop, resulting in shorter spans. As the child gets older, articulation improves, allowing more efficient and faster subvocal rehearsal, so that more items can be maintained in the loop.

Further evidence in support of this account comes from studies that have investigated the developmental course of the word length and acoustic similarity effects mentioned earlier. For example, for auditory presented items significant word length effects have been found from 4 years of age (Roodenrys, Hulme and Brown, 1993; Cowan, Keller, Hulme, Roodenrys, McDougall and Rack, 1994) and significant acoustic similarity effects from the same age (Hulme, 1987). Furthermore, these studies also found that the size of the word length and acoustic similarity effects increased with age. Two important conclusions are drawn from these findings. The first is that even fairly young children seem to engage in rehearsal based processes for the storage of verbal information and are sensitive to phonological information. The second is that the rehearsal and phonological discrimination processes improve with age.

The situation is slightly more complicated when the verbal items are presented visually as pictures of nameable objects. Hitch, Halliday, Schaafstel and Hefferman (1991) found word length effects and acoustic similarity effects in 5 year olds, but only if the items were explicitly named by either the experimenter or the child as the pictures were presented. If there was no naming then word length and acoustic similarity were only observed in the 11 year old group. Similarly Hulme (1987), in the study mentioned earlier found a small, but nevertheless significant effect of acoustic similarity in 4, 7 and 10 year old children, but no evidence of an increase in the size of the effect with age. Again, the pictures were named as they were presented to the child. The explanation for the delayed appearance of word length and acoustic similarity effects for visual presentation is attributed to the younger children failing to consistently recode the picture to its name. Therefore, there are no effects that rely on speech based processing, and the appearance of word length and acoustic similarity only occur when the pictures are named.

Research studies consistently show that children with Down's syndrome have characteristically poor short-term memory. For example, Mackenzie and Hulme (1987) compared groups of mental age matched typically developing chil- dren, a learning disabled group and a group with Down's syndrome. They found that on an auditory sequential memory task the group with Down's syndrome were poorer than the typically developing children and also poorer than the other severely learning disabled group. Furthermore, over a five year follow up the memory spans of the group with Down's syndrome did not improve as much as their increase in mental age would have predicted. That is, for the children with Down's syndrome there was an increasing difference between mental age and short-term memory performance.

Children and adults with Down's syndrome show poor auditory memory compared with visual memory and recognition memory (Marcell and Armstrong, 1982). As a population they also tend to have poor articulation skills (Dodd, 1976; Gibson, 1978). Given this combination of factors then it could be hypothesised that from a 'working memory' perspective they would be particularly disadvantaged in tasks that require use of the 'phonological loop'. In particular it was predicted that they would fail to show the standard effects that rely on a fully operational 'phonological loop' system: for example, little or no word length effect since they have poor articulation and therefore inefficient subvocal rehearsal; and show little or no acoustic similarity effect since they have poor auditory and phonological processing skills.

The primary aim of the present study was to assess the performance of children with Down's syndrome on a battery of working memory tasks and compare different age groups in order to see whether there are developmental changes in the word length and acoustic similarity effects in this population. The secondary aims were (i) to assess the relationship between these measures of memory and the children's phonological skill as measured by a rhyme processing task and (ii) assess the extent to which these children were influenced by the visual properties of the items to be recalled.

\section{Method}

\section{Participants}

A total of 62 children (30 male and 32 female), with Down's syndrome participated in the experiment; 22 under 7 year olds (mean age 5 years 9 months; range 4 years 4 months to 6 years 9 months), 207 to 11 year olds (mean age 9 years 0 months; range 7 years 4 months to 10 years 6 months) and 20 over 11 year olds (mean age 13 years 2 months; range 11 years 1 month to 18 years 1 month). The children were recruited from local schools in two different areas of the south of England. The schools included mainstream and special education establishments. The children came from a range of socio-economic backgrounds.

\section{Materials and Procedure}

Individual testing took place either at the child's home or at school. As part of a larger study (Broadley, 1994), a battery of memory and language based tasks were administered to each child, but the focus in this paper is on the following measures. All of the items and words used had been pretested with the children to ensure that they knew both the names of the pictures and that the names corresponded to those used by the experimenter. The sets were identical to those used in previous studies of working memory with young children (Hulme et al. 1987). 


\section{Auditory word span}

On each trial the experimenter spoke aloud a sequence of words taken from different sample sets. These sets varied in the word length of the items in the set; one syllable items (book, dog, car, pig, bus, cup), two syllable items (tractor, flower, rocket, table, apple), three syllable items (umbrella, banana, telephone, newspaper, elephant, kangaroo); acoustically similar (cat, hat, bat, man, rat, bag) and acoustically dissimilar (bus, kite, bell, book, car, pig). The words in each list were sampled randomly without replacement from the chosen set. The number of words to be memorised started with one word lists and increased across trials until the child failed to recall all the items in their correct position. There were three trials at each list length. The longest sequence recalled correctly was recorded as the word span.

\section{Visual word span}

The word sets used here were identical to those in the auditory condition. The stimuli were black and white line drawings of the words. On each trial the child was presented with a sequence of pictures shown by the experimenter. As each picture was presented it was simultaneously named by the experimenter and placed face down in front of the child. After the last item had been presented the child verbally recalled the list. The scoring, timing and sequencing was as for the auditory condition.

\section{Visual similarity}

The children were also presented with a set of black and white line drawings of words that had visually similar pictorial representations. The item lists were (ball, wheel, apple, orange), (spade, screw, fork, rake), (television, case, gate, box), (horse, goat, zebra, donkey), (car, ambulance, truck, bus). The presentation procedure was the same as that used in the Visual Word Span conditions. The score recorded was the longest list the child could recall correctly in order.

\section{Digit span}

Auditory and visual digit span was measured using the subtests of the British Ability Scales (Elliot et al, 1978). These were presented and scored in the standard fashion.

In addition to these Working Memory measures a further task was introduced to assess the children's phonological processing skills.

\section{Phonological awareness}

This was assessed by using a variant of the oddity test used by Bryant and Bradley (1985) for sound categorisation. Black and white line drawings of pictures were presented to the child in sets of triples. Two of the pictures had names that rhymed. The child's task was to pick out the picture with the non-rhyming name. One point was given for each correct trial. The maximum score on the test was eight.

\section{Results}

\section{Word length}

The mean values of word span were calculated for the auditory and visual conditions for each word length and are shown for each age group in Table 1. As can be seen, the span scores confirm the low level of memory performance of this group.

The data were analysed using a mixed four way analysis of variance, with between subject factors of Age and Sex and within subject factors of Presentation Modality and Word Length. There was no significant main effect for Sex and no significant interaction with any other factor. There was a significant effect for Age $(F(2,59)=27.84, p<0.001)$ with the anticipated finding of the older children having larger spans. There was a significant effect for Word Length $(F(2,118)=71.68, p<0.001)$ which showed that the children's mean span scores decreased with increasing size of the item length, the standard word length effect. There was no significant effect for Presentation Modality and no significant interactions.

\section{Acoustic similarity}

The mean span scores in each condition for each age group are shown in Table 2.

\begin{tabular}{|l|c|c|c|c|}
\hline Age group & \multicolumn{2}{|c|}{ Auditory } & \multicolumn{2}{c|}{ Visual } \\
\hline \multirow{3}{*}{$<7$} & AS & AD & AS & AD \\
\cline { 2 - 5 } $7-11$ & 1.27 & 1.27 & 1.23 & 1.55 \\
$>11$ & 1.75 & 2.20 & 1.65 & 2.05 \\
\hline
\end{tabular}

Table 2. Mean span scores for acoustically similar (AS) and dissimilar $(A D)$ words as a function of modality of presentation and age.

Again the data were analysed by a mixed four way analysis of variance. There was no significant main effect for Sex and no significant interaction with any other factor. There was a significant effect for Age $(F(2,59)=24.97, p<0.001)$ with the older children having larger spans. There was also a significant effect for Acoustic Similarity $(F(1,59)=30.12, p<0.001)$. This showed that the children's mean span scores were significantly larger in the acoustically dissimilar lists $($ mean $=2.0)$ than in the acoustically similar list (mean=1.65). There was no significant effect for Presentation Modality and no significant interactions.

\section{British Ability Digit Span}

The mean digit span scores in each condition for each age group are shown in Table 3 (page 6). The scoring of this test is done over two items at each list length, and a point given for each item correctly recalled. The mean score therefore is approximately double the length of list that the child could accurately recall.

The results of the three way analysis of variance showed again no significant main effect for Sex or any significant interaction with any other factors. There were significant 


\begin{tabular}{|l|c|c|}
\hline Age group & Auditory & Visual \\
\hline$<7$ & 1.91 & 2.82 \\
$7-11$ & 5.05 & 5.25 \\
$>11$ & 6.85 & 7.60 \\
\hline
\end{tabular}

Table 3. Mean digit span scores from the British Ability Scores as a function of modality of presentation and age.

effects for Age $(F(2,59)=15.33, p<0.001)$ with the older children having larger spans. There was also a significant effect for Presentation Modality $(F(1,59)=16.54, p<0.01)$ with the children scoring significantly higher in the visual condition (mean $=5.15$ ) than in the auditory condition (mean $=4.52$ ).

\section{Visual similarity}

The mean values for word span for the visually similar items are presented in Table 4, with the data from the 1 and 2 syllable visual word span conditions. Unfortunately when the visually similar lists were constructed they comprised items of 1 and 2 syllable duration. Since number of syllables was not controlled both of the word span conditions were used as comparison for the visually similar condition.

\begin{tabular}{|l|c|c|c|}
\hline Age group & Vis Simm & $\begin{array}{c}\text { Vis Diss } \\
(1 \text { syll })\end{array}$ & $\begin{array}{c}\text { Vis Diss } \\
(2 \text { syll })\end{array}$ \\
\hline$<7$ & 0.77 & 1.55 & 1.18 \\
$7-11$ & 1.50 & 2.05 & 1.70 \\
$>11$ & 1.70 & 2.40 & 2.20 \\
\hline
\end{tabular}

Table 4. Mean word span for visually similar (Vis Simm) and dissimilar (Vis Diss) pictures as a function of age.

The data were analysed using a mixed three way analysis of variance. As in the other analyses there was no significant main effect for Sex and no significant interaction with any other factors. There was a significant main effect for Age $(F(2,59)=19.87, p<0.001)$ such that the older children had larger spans. There was also a significant effect for Visual Similarity $(F(2,118)=36.0, p<0.001)$. This revealed that the children's span scores in the visually similar condition were lower (mean $=1.31$ ) than either of the dissimilar conditions (1.98 and 1.68 for the 1 and 2 syllable conditions respectively). There were no other significant effects.

\section{Rhyme judgements}

The mean rhyme scores for each age group are shown in Table 5. A one way analysis of variance showed that there were no significant differences between the three age groups $(F(2,59)=1.21, p>0.05)$. Most children scored very low on this task.

\begin{tabular}{|l|c|}
\hline Age group & Rhyme score \\
\hline$<7$ & 0.23 \\
$7-11$ & 0.70 \\
$>11$ & 0.95 \\
\hline
\end{tabular}

Table 5. Mean rhyme score as a function of age.
The correlations between the span scores and the rhyme oddity test are shown in Table 6 . As can be seen there is very little relationship between the word span scores and the rhyme scores. Only the correlation between the one syllable span measure is significantly related to rhyme judgement performance. However, the correlations between rhyme judgement and the auditory span measures are all higher than those between the visual word span measures and rhyme judgement. Thus it does appear to be sensitive to verbal versus pictorial presentation and therefore a measure of phonological processing, albeit a rather weak one.

Table 6. Correlation of rhyme judgements with auditory and visual word span

\begin{tabular}{|c|c|c|c|}
\hline 1 - syllable & 2 - syllable & 3 - syllable & BAS dig: \\
\hline $0.39^{*}$ & 0.32 & 0.26 & $0.42^{\star *}$ \\
\hline 0.17 & 0.23 & 0.21 & $0.35^{*}$ \\
\hline
\end{tabular}

\section{Discussion}

The issues raised in the introduction to the paper were the extent to which the short-term memory performance of children with Down's syndrome could be incorporated within the theoretical framework of working memory. While the present results show that certain aspects of their performance can be explained by the operation of a phonological loop, the data also show that verbal memory span relies on more than speech based coding.

The children tested here showed that word length had a small but consistently significant effect on memory for both words and pictures for all three age groups. Moreover there was no change in this word length effect with age. The standard interpretation of this would be that these children were using a subvocal rehearsal based mechanism for storing and recalling the items. As there was also no difference dependent on whether the items were presented as speech or pictures, this would suggest equivalent use of a speech code for memorising pictures. There was no indication that the children might have been using any extra visual coding (Paivio, 1971) in the picture presentation conditions. For the auditory presentation these data support the previous research which has found consistent word length effects from approximately 4 years of age and no change with age (Hitch et al., 1989, 1991; Roodenrys et al. 1993). The data also support the findings of Hulme et al. (1987), who found a word length effect, for pictorial presentation, in children as young as 4 years old, but only when the items were named at presentation. This was also the procedure adopted here. Hence the pattern of results so far is consistent with an articulatory loop based account of storage and retrieval in this group of subjects.

Further support for the hypothesis that these children were using speech based coding to store the items comes from the acoustic similarity data. There was again a small but significant effect with smaller spans found for acoustically similar lists than acoustically dissimilar lists. As in the word length data there was no evidence for differential use of a speech based code in the auditory as opposed to the visual presentation conditions. The acoustic similarity effect was equivalent across both modalities. There was also no change in the size of the acoustic similarity effect with age. This contrasts 
with the findings from studies of normally developing children. Hulme (1987), using similar materials and procedures to those used here, found that acoustic similarity had more effect in older children in an auditory presentation condition, but no change with age in a visual presentation condition. This implies more reliance on speech based coding with increasing age. Ford and Silber (1994) also found a more pronounced acoustic similarity effect in older children. Both sets of researchers attribute this to increasing use of rehearsal in the older children. Thus span performance is sensitive to both the efficiency of phonological coding and use of subvocal rehearsal.

The most surprising aspect of the data reported here is that the pattern of results from this group is by and large very similar to that obtained from typically developing children. It is obvious that this group of children with Down's syndrome have much smaller spans, and that they show less of an increase with age than normally developing children, but otherwise their data show the same effects. On the surface this contradicts the hypotheses outlined earlier, where it was anticipated that given their poor articulation and auditory memory deficits there would be no consistent word length or acoustic similarity effects and that performance would be better in the visual modality. The results also contrast with those found by Raine et al. (1991) who found no effect for word length in a group of speech-disordered children, which was again taken as evidence for memory span being dependent on a process of subvocal rehearsal.

The question of what form of coding the children used in the tasks carried out here must include the possibility that visual memory mechanisms were also used. Two pieces of evidence support this idea. The first is the lack of any difference dependent on the modality of presentation. In the studies that have directly compared visual and auditory presentation in typically developing children it has been found that memory recall is poorer under visual presentation than auditory (Hulme, 1987). The argument is that recoding the visual stimulus to a verbal form takes up processing resources. This does not occur in the auditory condition. For the children in this study, since there was no difference between vision and audition, it suggests that they were able to use the visual information to compensate for their poorer auditory information. The second piece of evidence is the data from the visual similarity conditions. The fact that the children were consistently poorer on the visually similar lists, in comparison with the dissimilar lists would support the hypothesis that they were using visual information in the encoding and storing of the items. A similar kind of effect has been found with typically developing children.

In the Hulme (1987) study, children from 4 to 10 years old remembered more acoustically similar items when they were visually presented (and labelled) than when they were spoken. The children were using the information (visual) that discriminated between the items. It is assumed that typically developing children increasingly use verbal recoding for remembering sequences of pictures. It is equally possible that the converse might also take place and that children use visual information, perhaps images for remembering verbally presented lists. Given that the child with Down's syndrome is reasoned to have relatively better developed visual processing skills, it is entirely possible that the lack of modality differences found here reflect two compensatory processes - use of visual information in the visual presenta- tion condition and verbal to visual recoding in the auditory presentation condition. Further research is needed to elucidate these encoding mechanisms and strategies.

In summary then the evidence from this study supports the view that children with Down's syndrome are susceptible to both word length and acoustic similarity effects in the storage and recall of verbal items. If these phenomena reflect the operation of rehearsal and speech based coding then it suggests some possibility of remediation of the very poor memory spans shown by these children. There is already some evidence that the use of rehearsal based training programmes can lead to improved short-term memory performance in this group of children (Belmont, Butterfield and Borkowski, 1978; Hulme and Mackenzie, 1992; Broadley and MacDonald, 1993). The question of whether phonologically based training could, either on its own or in conjunction with rehearsal, lead to similar improvements needs to be tested. Therapy that alleviated these memory deficits might also be of benefit for other aspects of cognitive functioning and development, given the widely held view that short-term memory functions as a working memory system that is involved in a variety of everyday cognitive activities such as language processing, reading and reasoning.

\section{References}

Baddeley,A.D. (1986). Working Memory. Oxford: Oxford University Press.

Baddeley,A.D., and Hitch,G. (1974) Working memory. In G. $\mathrm{H}$. Bower (Ed.), The Psychology of Learning and Motivation (Vol. 8, pp. 47-90). New York: Academic Press

Baddeley,A.D., Thomson,N., and Buchanan,M. (1975) Word length and the structure of short-term memory. Journal of Verbal Learning and Verbal Behavior, 14, 575-589.

Belmont,J.M., Butterfield,C.E. and Borkowski,J.G. (1978) Training retarded people to generalise memorisation methods across memory tasks. In M.M. Grunneberg, P.E. Morris and R.N. Skyes (Eds.) Practical Aspects of Memory. New York: Academic Press.

Broadley, I.W. (1994) Teaching Short-term Memory Skills to Children With Down's Syndrome. (Unpublished PhD thesis, University of Portsmouth, Portsmouth, UK).

Broadley, I.W. and MacDonald, J. (1993) Teaching shortterm memory skills to children with Down's syndrome.Down's Syndrome: Research and Practice, 1, 56-62.

Bryant,P.E. and Bradley,L.(1985)Children's Reading Problems. Oxford: Blackwell.

Chi,M. (1977) Age differences in memory span. Journal of Experimental Child Psychology, 23, 226-281.

Cowan,N., Keller,T.A., Hulme,C., Roodenrys,S., McDougall,S. and Rack,J. (1994) Verbal memory span in children: Speech timing clues to the mechanisms underlying age and word length effects. Journal of Memory and Language, 33, 234-250.

Dodd,B. (1976) A comparison of the phonological systems of mental age matched normal, severely subnormal and Down's syndrome children. British Journal of Disorders of Communication, 11, 27-123.

Elliot,C.D., Murray,D.J., and Pearson,L.S. (1978) British Ability Scales. Windsor: NFER-Nelson.

Ford,S. and Silber,K.P. (1994) Working memory in children: A developmental approach to the phonological coding of pictorial material. British Journal of Developmental Psychology, 12, 165-175. 
Gathercole,S.E. and Baddeley,A.D. (1989) Evaluation of the role of phonological STM in the development of vocabulary in children: A longitudinal study. Journal of Memory and Language, 28, 200-213.

Gibson,D. (1978) Down's Syndrome: The Psychology of Mongolism. Cambridge: Cambridge University Press.

Hitch,G.J. and Halliday,M.S. (1983) Working memory in children. Philosophical Transactions of the Royal Society, Series B, 302, 324-340.

Hitch,G.J., Halliday,M.S., Dodd,A., and Littler,J.E. (1989) Development of rehearsal in short-term memory: Differences between pictorial and spoken stimuli. British Journal of Developmental Psychology, 7, 347-362.

Hitch,G.J., Halliday,M.S., Schaafstel,A.M., and Hefferman,T.M. (1991). Speech, "inner speech", and the development of short-term memory: Effects of picture-labelling on recall. Journal of Experimental Child Psychology, 51, 220-234.

Hulme,C. (1987) The effects of acoustic similarity on memory in children: A comparison between visual and auditory presentation. Applied Cognitive Psychology, 1, 45-52.

Hulme,C., and Mackenzie,S. (1992) Working Memory and Severe Learning Difficulties. Hove: Lawrence Erlbaum Associates.

Hulme,C., Silvester, J., Smith,S., and Muir,C. (1987) The effects of word-length on memory for pictures: Evidence for speech coding in young children. Journal of Experimental Child Psychology, 41, 61-75.

Mackenzie,S., and Hulme,C. (1987) Memory span development in Down's syndrome, severely subnormal and normal subjects. Cognitive Neuropsychology, 4 (3), 303-319.
Marcell,M.M., and Armstrong,V. (1982) Auditory and visual sequential memory of Down Syndrome and non-retarded children. American Journal of Mental Deficiency, 87 (1), 8695.

Paivio, A. (1971) Imagery and Verbal Processes. New York: Holt, Rinehart and Winston.

Raine,A., Hulme,C., Chadderton,H., \& Bailey,P. (1991) Verbal short-term memory span in speech disordered children: Implications for articulatory coding in short-term memory. Child Development, 62, 415-423.

Roodenrys,S., Hulme,C. and Brown,G. (1993) The development of short-term memory span: Separable effects of speech rate and long-term memory. Journal of Experimental Child Psychology, 56, 431-442.

Salamé,P. and Baddeley,A.D. (1986) Phonological factors in STM: Similarity and the unattended speech effect.Bulletin of the Psychonomic Society, 24, 263-265.

Address for correspondence:

Dr J. MacDonald, Department of Psychology,

University of Portsmouth,

King Charles Street,

Portsmouth, PO1 2ER U.K.

\section{References cited in the Editorial on page 2}

Broadley,I. and MacDonald,J. (1993). Teaching short term memory skills to children with Down's syndrome.Down's Syndrome: Research and Practice, 1 (2), 56-62.

Broadley,I., MacDonald,J. and Buckley,S. (1994). Are children with Down's syndrome able to maintain skills learned from a short-term memory training programme? Down's Syndrome: Research and Practice, 2 (3), 116-122.
Buckley,S. (1993). Developing the speech and language skills of teenagers with Down's syndrome. Down's Syndrome: Research and Practice, 1 (2), 6371).

Hulme,C. (1987). The effects of acoustic similarity on memory in children: A comparison between visual and auditory presentation. Applied Cognitive Psychology, 1, 45-52. 